Casos Clínicos

Arch. Esp. Urol., 61, 7 (828-831), 2008

\section{ADENOCARCINOMA VESICAL. APORTACIÓN DE UN CASO CLÍNICO}

Mário Jorge Soares, Tiago Neves, Ana Covita, Pedro Monteiro, Artur Canhoto, Rui Nogueira, José Luis Barreto, José Carlos Mendonça, Manuela Maya' y Helder Monteiro.

Servicio de Urología y Servicio de Anatomía Patológica. Hospital Egaz Moniz. Lisboa. Portugal.

Resumen.- OBJETIVO: El adenocarcinoma primario de vejiga es una entidad rara. Realizamos una revisión de la literatura y presentamos un caso clínico.

MÉTODOS/RESULTADOS: Se trata de una paciente del sexo femenino de 74 años de edad que acude al servicio de urgencias por hematuria macroscópica, sin otra sintomatología acompañante. Se sometió a varios exámenes auxiliares de diagnóstico, incluyendo ecografía renal y vesical, cistoscopia y TAC abdominal y pélvico. Después de la realización de resección transuretral de pólipo vesical y frente al diagnóstico de adenocarcinoma músculo invasivo, se somete a una evaluación ginecológica completa, excluyendo enfermedad ginecológica. Se procede a exanteración pélvica anterior. El informe anatomo patológico muestra un adenocarcinoma vesical primario, pT3aNO, con ganglios linfáticos regionales negativos. Después de 6 meses de seguimiento, la paciente no presenta complicaciones resultantes de la cirugía o evidencia de evolución de su enfermedad.

CONCLUSIÓN: El Adenocarcinoma vesical primario es una entidad rara. Al contrario del carcinoma urotelial, responde mal a la quimioterapia y radioterapia, por lo que el único tratamiento eficaz es la cirugía radical.

Palabras clave: Tumor vesical. Adenocarcinoma primario.

Summary.- OBJECTIVE: Primary bladder adenocarcino$m a$ is a rare entity. We performed a review of the literature on this subject and present a clinical case.

METHOD/RESULTS: A 74 year old female patient presents to the emergency department with complaints of gross hematúria not accompanied by lower urinary tract symptoms. Diagnostic work-up included renal and bladder ultrasound, cistoscopy and abdomino-pelvic C. T. scan. Metastatic disease was excluded. Trans-urethral resection revealed an invasive adenocarcinoma. A gynaecologic origin was excluded by further gynaecologic examination and an anterior pelvic exanteration was performed. The bladder specimen showed primary bladder adenocarcinoma, pT3aNO. At 6 months of follow-up, the patient does not present disease progression or surgical complications.

CONCLUSION: Primary bladder adenocarcinoma is rare. Unlike urothelial carcinoma, it responds poorly to chemotherapy or radiotherapy. Radical Cistectomy offer the best chance of long-term survival.

Keywords: Bladder tumor. Primary adenocarcinoma.

\section{CASO CLÍNICO}

Paciente del sexo femenino de 74 años de edad que acude al servicio de urgencias por hematuria macroscópica, sin otra sintomatología acompañante. Antecedentes médicos y quirúrgicos irrelevantes. Ex-fumadora. La ecografía renal y vesical revela una ectasia pielocalicial moderada del riñón derecho y una masa sólida, sésil, localizada en la pared lateral derecha endovesical de $4 \mathrm{~cm}$ de diámetro. La cistoscopia (se realiza en el mismo acto quirúrgico) muestra un tumor sésil de 3,5 


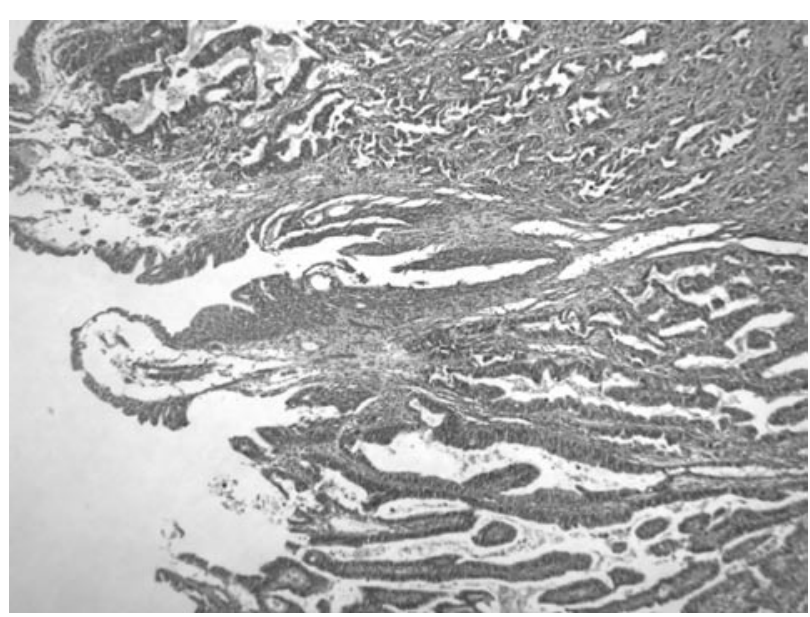

FIGURA 1. Adenocarcinoma vesical.

cm localizado en la pared lateral derecha. La palpación bi-manual no existe fijación. La biopsia del tumor revela adenocarcinoma invasivo, no excluyendo origen ginecológico, por lo que se realiza examen ginecológico completo que se revela negativo. El estudio de extensión no muestra metástasis. Se le efectúa exanteración pélvica anterior y ureterostomia cutánea trans-ileal tipo Bricker. El estudio histológico describe un adenocarcinoma de patrón en parte papilar en parte túbulo-quístico de alto grado citológico, pT3aNO (Figuras 1, 2 y 3).

La paciente tuvo una recuperación satisfactoria, sin complicaciones per-operatorias. A los 6 meses de seguimiento pos-operatorio no revela evolución clínica ni en la imagen de la neoplasia o de complicaciones resultantes de la cirugía.

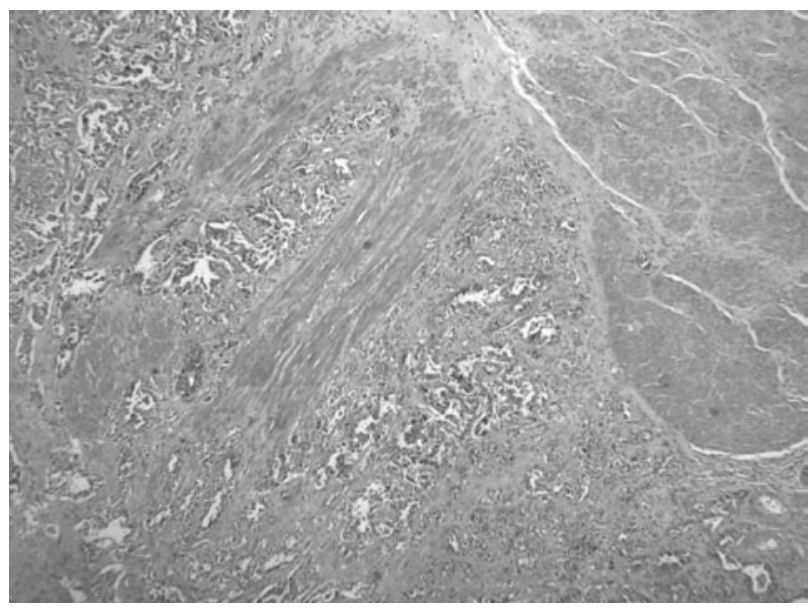

FIGURA 2. Infiltración por el adenocarcinoma con disociación de fibras musculares.

\section{DISCUSIÓN}

El adenocarcinoma vesical primario representa el 0,5$2 \%$ de todos los tumores vesicales, siendo el tercer tipo mas frecuente de los tumores epiteliales de la vejiga (2). No obstante, muchos urólogos no encuentran este tipo de tumor durante toda su vida profesional. Constituye el tipo histológico mas frecuente en pacientes con extrofia vesical, teniendo éstos la probabilidad de padecer este tipo de tumores durante toda la vida de $4 \%(3)$. Aparece, con mayor frecuencia, en regiones geográficas donde la infección por shistosoma es endémica, representando el $10 \%$ de los tumores epiteliales de la vejiga en estas regiones (4).

No se identifican factores de riesgo dignos de mención. Según Mostofi y cols. (5), el potencial metastático del urotelio sigue dos patrones distintos. Ambos tendrían como factor causal la irritación y la infección vesical crónica, provocando por consecuencia metaplasia intestinal del urotelio. El primero implicaría la formación de quistes a partir de los nidos de Von Brunn (cistitis quística). Con la transformación de las células de revestimiento de estas en células columnares productoras de moco aparecería la cistitis glandular, tenida como lesión pre-maligna. En alternativa, el segundo patrón ocurre con metaplasia intestinal sin la invaginación epitelial para la lámina propia (nidos de Von Brunn). Esta teoría fue respondida recientemente. Corica y cols.. (6) hizo una revisión de 53 casos de pacientes con extrofia vesical y metaplasia intestinal de la mucosa vesical. Ningún paciente desenvuelve adenocarcinoma vesical en un tiempo de seguimiento medio de 13 años.

La presentación clínica es idéntica a las otras neoplasias vesicales predominando la hematuria macroscópica y síntomas urinarios irritativos.

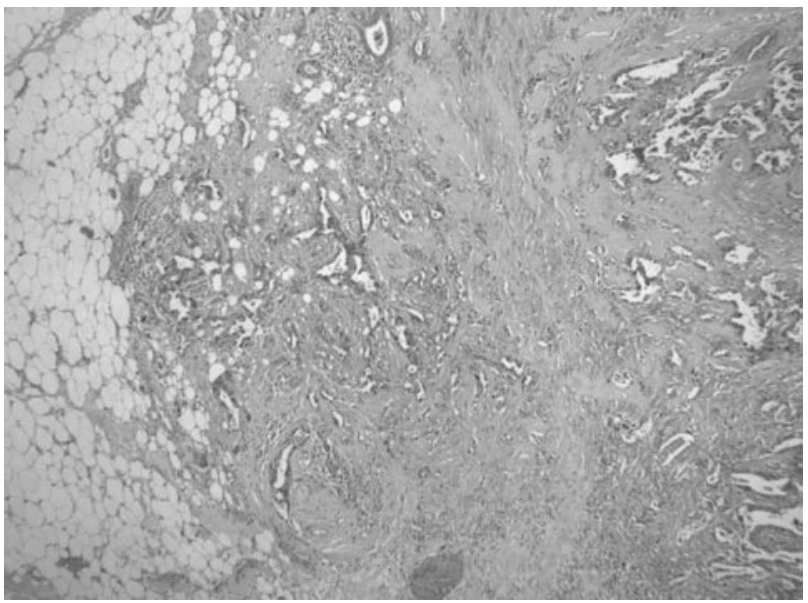

FIGURA 3. Invasión de la grasa. 
La cistoscopia puede presentarse como una lesión papilar o una lesión achatada, infiltrativa con edema de la mucosa que la recubre. La mayoría de los pacientes tienen enfermedad músculo invasiva (7). Los diagnósticos diferenciales son adenocarcinoma de próstata, adenocarcinoma colo-rectal, neoplasia de ovario y otras neoplasias ginecológicas, envolviendo la vejiga por metástasis o por extensión directa.

Adenocarcinoma vesical se clasifica en 3 categorías, conforme a su origen: primario, del uraco y metastático (8). El diagnóstico diferencial puede ser difícil, pero una anamnesis y un examen objetivo cuidadoso asociados a técnicas inmunohistoquímicas permiten un diagnóstico cierto, en la mayoría de los casos.

La distinción entre adenocarcinoma primario y de uraco es importante por cuestiones pronosticas y terapéuticas. Algunos autores definieron prerrequisitos para el diagnóstico de adenocarcinoma del uraco:

1. Localización en la cúpula vesical,

2. Ausencia de cistitis quística o glandular,

3. Envolviendo predominantemente a la muscular en vez de la submucosa con un epitelio de transición intacto o ulcerado,

4. Demostración de un resquicio del uraco unido al tumory

5. Presencia de una masa neoplásica suprapúbica (8).

El carcinoma de próstata se diferencia a través del PSA y el tacto rectal. En el caso del adenocarcinoma colorectal aparece con signos y síntomas relacionados con lesión intestinal primaria (melenas, rectorragias, alteraciones de los hábitos intestinales y la oclusión intestinal). La invasión vesical por el carcinoma de endometrio es un fenómeno tardío en la evolución de la enfermedad y el sangrado vaginal lo precede. El carcinoma del cuello uterino puede invadir la vejiga y ser confundido por una adenocarcinoma vesical primario. El examen ginecológico, ecografía endovaginal, colposcopia con biopsia ayudarán a realizar el diagnóstico diferencial de ambos, tal y como harán con la neoplasia del ovario que con sus patrones histológicos variados podrá llevar a error.

La utilización de técnicas de inmunohistoquímica para auxiliar el diagnóstico histológico se ha demostrado decisivo. La vimentina ayudará a identificar al carcinoma de endometrio. De igual modo varias inmunoestatinas, como la citoqueratina-7 y -20, trombomodulina y B-catenina tiene valor para el diagnóstico diferencial del adenocarcinoma colo-rectal (9).

En términos histopatológicos, el adenocarcinoma vesical primario se clasifica como carcinoma glandular, carcinoma coloide, adenocarcinoma papilar, carcinoma de cé- lulas en anillo de sinete (que macroscópicamente puede dar origen a un tumor infiltrativo tipo linitis plástical y carcinoma de células claras (10). No ha sido atribuido valor pronóstico a esta subclasificación histológica, a excepción del tipo con células en anillo de sinete, que según algunos autores parece tener peor pronóstico (11).

No existen datos suficientes para tomar conclusiones sólidas sobre el tratamiento del adenocarcinoma vesical. Siendo una entidad rara, el número de pacientes presentados en varios estudios es limitado. La resección transuretral asociada o no a radioterapia (12) y la cistectomía parcial para enfermedad localizada en las partes móviles de la vejiga (13) tienen ambas resultados poco satisfactorios. El único tratamiento que ofrece la posibilidad de cura es la cistectomía radical $(15,16)$. El-Mekresh y cols. (3) publicó la mayor serie de pacientes tratados por adenocarcinoma vesical primario; 185 pacientes fueron tratados con cistectomía radical con una esperanza de vida de $55 \%$ a los 5 años. La esperanza de vida se correlaciona con el estadio, grado y la invasión ganglionar. Es necesario tener en cuenta que se trata de pacientes de una zona geográfica donde la infección por shistosoma es endémica y según algunos autores los adenocarcinomas primarios en estas zonas son siempre de menor grado histológico.

La radioterapia externa está asociada a pobres resultados. En una serie de Gill y cols. (14), la esperanza de vida media fue de 13 meses. En otro estudio, la esperanza de vida a los 5 años fue de $20 \%$ (10).

La utilización del 5-fluorouracilo, tanto en monoterapia como en regímenes de varios quimioterápicos han sido utilizada en algunos casos de tumores irresecables a través de infusión intra-arterial con alguna respuesta objetiva. El impacto sobre la esperanza de vida no fue claramente establecido (17).

\section{CONCLUSIÓN}

El adenocarcinoma vesical es poco frecuente y los datos publicados son escasos e insuficientes para sacar conclusiones en cuanto a su tratamiento. No obstante, parece adecuado afirmar que la radioterapia es ineficaz, la resección endoscópica es la cistectomía parcial están asociadas a una esperanza de vida muy reducida, la única curación es la cistectomía radical.

\section{BIBLIOGRAFIA y LECTURAS RECOMENDADAS (*lectura de interés $y^{* *}$ lectura fundamental)}

**1. ABOL-ENEIN, H.; KAVA, B.R.; CARMACK, A.J.: "Nonurothelial Cancer of the Bladder". Urology, 69: 93, 2007. 
*2. THOMAS, D.G.; WARD, A.M.; WILLIAMS, J.L.: “A study of 52 cases of adenocarcinoma of the bladder". Br. J. Urol., 43: 4, 1971.

3. SMEULDERS, N.; WOODHOUSE, C.R.: "Neoplasia in adult extrophy patients". BJU Int., 87: 623, 2001.

*4. EL-MEKRESH, M.M.; EL-BAZ, M.A.; ABOLENEIN, H. y cols.: "Primary adenocarcinoma of the urinary bladder: A report of 185 cases". Br. J. Urol., 82: 206, 1998.

5. MOSTOFI, F.K.; THOMSON, R.V.; DEAN, A.L.: "Mucous adenocarcinoma of the urinary bladder". Cancer, 8: 741, 1995.

6. CORICA, F.A.; HUSMANN, D.A.; CHURCHILL, B.M. y cols.: "Intestinal metaplasia is not a strong risk factor for bladder cancer: A study of 53 cases with longterm follow-up". Urology, 50: 427, 1997.

7. EL-BOLKAINY, M.N.; MOKHTAR, N.M.; GHONEIM, M.A. y cols.: "The impact of schistosomiasis on the pathology of bladder cancer". Cancer, 48: 2643, 1981.

8. WHEELER, J.D.; HILL, W.T.: "Adenocarcinoma involving the urinary bladder". Cancer, 7: 119, 1951.

9. WANG, H.L.; LU, D.W.; YERIAN, L.M. y cols.: "Immunohistochemical distinction between primary adenocarcinoma of the urinary bladder and secondary colorectal adenocarcinoma”. Am. J. Surg. Pathol., 25: 1380, 2001.

10. ANDERSTROM, C.; JOHANSSON, S.L.; VON SCHULTZ, L.: "Primary adenocarcinoma of the urinary bladder. A clinicopathologic and prognostic study". Cancer, 52: 1273, 1983.

11. GRIGNON, D.J.; RO, J.Y.; AYALA, A.G. y cols.: "Primary adenocarcinoma of the urinary bladder. A clinicopathological analysis of 72 cases". Cancer, 67: 2165, 1991.

12. MALEK, R.S.; ROSEN, J.; O`DEA, M.: "Adenocarcinoma of the bladder". Urology, 21: 357, 1983.

13. ABENOZA, P.; MONIVEL, C.; FRALEY, E.: "Primary adenocarcinoma of the urinary bladder. A clinico-pathological study of 16 cases". Urology, 31: 9, 1987.

14. GILL, H.S.; DHILLON, H.K.; WOODHOUSE, C.R.: "Adenocarcinoma of the urinary bladder". Br. J. Urol., 64: 138, 1989.

15. WILSON, T.G.; PRITCHETT, T.R.; LIESKOVSKY, G. y cols.: Urology, 38: 223, 1991.

16. GHONEIM, M.A.; EL-MEKRESH, M.M.; EL-BAZ, M.A. y cols.: "Radical cistectomy for carcinoma of the bladder: Critical evaluation of the results in $1.026 \mathrm{ca}-$ ses". J. Urol., 158: 393, 1997.

17. AWAKURA, Y.; YAMAMOTO, M.; FUKUZAWA, S. y cols.: "Intra-arterial infusion of 5-fluorouracil, leucovorin and cisplatin for primary adenocarcinoma of the urinary bladder". Int. J. Urol., 6: 581, 1999. 